\title{
Minimization of Quadratic Binary Functional with Additive Connection Matrix
}

\author{
Leonid Litinskii \\ Center of Optical Neural Technologies Scientific Research Institute for System Analysis, \\ Russian Academy of Sciences, \\ 44/2 Vavilov Str, Moscow 119333, Russia. \\ litin@mail.ru
}

\begin{abstract}
N \times N)$-matrix is called additive when its elements are pair-wise sums of $N$ real numbers $a_{i}$. For a quadratic binary functional with an additive connection matrix we succeeded in finding the global minimum expressing it through external parameters of the problem. Computer simulations show that energy surface of a quadratic binary functional with an additive matrix is complicate enough.
\end{abstract}

\section{Introduction}

In the present paper we analyze the classic problem of discrete mathematics that is minimization of a quadratic functional depending on the great number $N$ of binary variables $s_{i}$ :

$$
E(\mathbf{s})=-\frac{(\mathbf{J} \mathbf{s}, \mathbf{s})}{2 N}=-\frac{1}{2 N} \sum_{i, j=1}^{N} J_{i j} s_{i} s_{j} \longrightarrow \min , s_{i}= \pm 1 .
$$

This problem arises in a lot of scientific fields of knowledge beginning from physics of magnetic materials and neural networks up to analysis of results of physical experiments and logistics. Usually the connection matrix $\mathbf{J}=\left(J_{i j}\right)_{1}^{N}$ is supposed to be symmetric one with zero diagonal elements: $J_{i j}=J_{j i}, J_{i i}=0$. The state of the system as a whole is given by $N$-dimensional vector $\mathbf{s}=\left(s_{1}, s_{2}, \ldots, s_{N}\right)$. Such vectors will be called configuration vectors or simply configurations. The characteristic $E(\mathbf{s})$ that has to be minimized will be called the energy of the state, and the configuration providing the global minimum of the functional (1) will be called the ground state.

In general the number of local minima of the functional (1) is exponentially large. Practically all minimization algorithms guarantee finding of a local minimum only. The exceptions are very rare and, as a rule, they are relied on specific properties of the connection matrix [1], [2]. The most widespread is the random minimization [1]. According this algorithm the spin dynamics is started from a random configuration. In randomized order the states of dissatisfied spins are changed. As a result the dynamic system step by step falls into the nearest local minimum. We used just the random minimization in our computer simulations (Section 3).

Very little is known about properties of the energy surface of the functional (1), namely, about the number and the structure of the set of local minima, about the ground 
state and the probability to find it and so on. In fact there is only one nontrivial connection matrix for which the ground state of the functional (1) can be indicated exactly. This is the Hebb matrix in the case when the value of the loading parameter $\alpha=M / N$ is small: $\alpha<0.07$ [3]. Then the global minimum of the functional (1) is achieved at any of $M$ random patterns.

Due to discrete character of the problem its theoretical analysis is very rare. From recent results let us point out the papers [4], [5], where the authors succeeded in connecting the depth of the local minimum with the probability of its random finding, and also described some characteristics of the energy surface.

In our work we introduce a class of additive matrices whose elements are pair-wise sums of a set of predetermined numbers $a_{i}$ :

$$
J_{i j}=\left(1-\delta_{i j}\right)\left(a_{i}+a_{j}\right), \quad i, j=1, \ldots, N, \quad \text { where } \quad\left\{a_{i}\right\}_{1}^{N} \in \mathbf{R}^{\mathbf{1}}
$$

and $\delta_{i j}$ is the Kronecker delta symbol.

The additive matrices generalize a special class of Hebb's matrices analyzed in [6]. For the functional (1) with the connection matrix (2) the ground state can be obtained exactly. We succeeded in presentation additive matrices in the form when the dependence of the ground state on external parameters of the problem can be described analytically. When the ground state is known, interesting results can be obtained with the aid of computer simulation. In the next Section we present the theory relating to the problem. In Section 3 we give the results of computer simulations.

\section{The Ground State}

1. It can be verified directly that for an additive matrix (2) the value of the functional (1) is equal to

$$
E(\mathbf{s})=\frac{(\mathbf{e}, \mathbf{a})-(\mathbf{s}, \mathbf{e})(\mathbf{s}, \mathbf{a})}{N}
$$

Here $\mathbf{a}=\left(a_{1}, a_{2}, . ., a_{N}\right)$ is $N$-dimensional vector whose coordinates are $a_{i}$, and $\mathbf{e}=$ $(1,1, \ldots, 1)$ is the "bisector" of the principal orthant of the space $\mathbf{R}^{\mathbf{N}}$. From minimization of the functional (3) one can pass to maximization of the functional

$$
F(\mathbf{s})=(\mathbf{s}, \mathbf{e})(\mathbf{s}, \mathbf{a}) \longrightarrow \max
$$

Let us denote by $\Sigma_{k}$ the class of all configurations s for which exactly $k$ coordinates are equal "-1":

$$
\Sigma_{k}=\{\mathbf{s}:(\mathbf{s}, \mathbf{e})=N-2 k\}, k=0,1, \ldots, N
$$

The class $\Sigma_{k}$ consists of $C_{k}^{N}$ configurations. For all these configurations the first multiplier in the expression (4) takes the same value $N-2 k$. Consequently, to maximize (4) among configurations from the class $\Sigma_{k}$, it is sufficient to find a vector $\mathbf{s} \in \Sigma_{k}$ maximizing the scalar product (s, a). This problem is not so difficult (see item 3 ). 
2. Suppose, we can find the vector $\mathbf{s}$ maximizing the scalar product $(\mathbf{s}, \mathbf{a})$ in the class $\Sigma_{k}$. Let us denote this vector as $\mathbf{s}(k)$, and let the value of the functional (4) for this vector be $F_{k}=F(\mathbf{s}(k))$ :

$$
F_{k}=(N-2 k)(\mathbf{s}(k), \mathbf{a})=\max _{\mathbf{s} \in \Sigma_{k}} F(\mathbf{s}), \quad k=0,1, . ., N .
$$

When finding all these vectors $\mathbf{s}(k)(k=0,1, . ., N)$, it is easy to find the global maximum of the functional (4), since the functional reaches its maximal value on one of the vectors $\mathbf{s}(k)$.

Note we do not need to compare between themselves all $N+1$ numbers $F_{k}$, but the first half of them only. The reason is that for any $k$ the classes $\Sigma_{k}$ and $\Sigma_{N-k}$ are inversion of each other: $\Sigma_{N-k}=-\Sigma_{k}$. Since for any configuration $\mathbf{s}$ the equality $F(\mathbf{s})=F(-\mathbf{s})$ is fulfilled, we obtain that $F_{k}=F_{N-k}$ for all values of $k$. Combining the cases of even and odd $N$ in one formula we obtain that to find the global maximum of the functional (4) it is necessary to find the largest of the values $F_{k}$, when $k \leq n=$ $[N / 2]$ :

$$
F_{0}, F_{1}, . ., F_{n}, n=\left[\frac{N}{2}\right] .
$$

3. Without loss of generality the numbers $\left\{a_{i}\right\}_{1}^{N}$ can be put in order according their increase:

$$
a_{1}<a_{2}<\ldots<a_{N} .
$$

Let us take any $k \leq n$. It is easy to see that the scalar product $(\mathbf{s}, \mathbf{a})$ reaches its maximum inside the class $\Sigma_{k}$ when the configuration vector is

$$
\mathbf{s}(k)=(\underbrace{-1,-1, \ldots,-1}_{k}, 1,1, . .1) .
$$

Indeed,

$$
(\mathbf{s}(k), \mathbf{a})=-\sum_{i=1}^{k} a_{i}+\sum_{i=k+1}^{N} a_{i}=\hat{a}-2 \hat{a}_{k}
$$

where

$$
\hat{a}=\sum_{i=1}^{N} a_{i}, \hat{a}_{k}=\sum_{i=1}^{k} a_{i}, \text { and } \hat{a}_{0}=0 .
$$

Let $\mathbf{s}$ be another configuration vector from the class $\Sigma_{k}$ for which numbers of negative coordinates $j_{1}<j_{2}<\ldots<j_{k}$ dose not take the first places. The scalar product $(\mathbf{s}, \mathbf{a})$ is equal to $(\mathbf{s}, \mathbf{a})=\hat{a}-2 \sum_{i=1}^{k} a_{j_{i}}$, and inequality $(\mathbf{s}(k), \mathbf{a})>(\mathbf{s}, \mathbf{a})$ is fulfilled since $\sum_{i=1}^{k} a_{i}<\sum_{i=1}^{k} a_{j_{i}}$ for any set of indices $\left\{j_{1}, j_{2}, \ldots, j_{k}\right\}$ that differs from $\{1,2, \ldots, k\}$.

Thus, under the condition of ordering (5), to find the global minimum of the functional (3) it is necessary to find the largest among the numbers

$$
F_{k}=(N-2 k)\left(\hat{a}-2 \hat{a}_{k}\right), k=0,1, . ., n=\left[\frac{N}{2}\right],
$$


where $\hat{a}$ and $\hat{a}_{k}$ are given in Eq.(7).

4. The initial problem (1)-(2) can be considered as solved: the expressions (6) restrict the set of configurations among which the ground state of the functional (2) should be found. To define which configuration is the ground state it is necessary to calculate $n$ numbers (8) and find the largest among them. It reminds unclear under which conditions this or that configuration (6) would be the ground state. If any of them will be the ground state or not? It turned out that these questions can be answered.

Without loss of generality let us suppose that the numbers $a_{i}$ have a special form:

$$
a_{i}=\alpha_{i}-t, \quad \alpha_{i} \in[0,1], t \geq 0 .
$$

In this presentation the values $\alpha_{i}$ are positive numbers from the unit interval, and the positive parameter $t$ can take an arbitrary value. It is not difficult to see that from the point of view of minimization of our functional an arbitrary set of numbers $a_{i}$ can be reduced to the form (9). For example, let us suppose that $a_{1}<\ldots<a_{N}<0$ and $a_{N}-a_{1} \leq 1$. Then we set $\alpha_{i}=a_{i}-a_{1}$ and $t=\left|a_{1}\right|$. This means that the numbers $a_{i}$ have the form (9). On the contrary, let the initial numbers $\tilde{a}_{i}$ have different signs and take on arbitrary values: $\tilde{a}_{1}<\ldots 0<\ldots<\tilde{a}_{N}$, and $\tilde{a}=\max \left(\left|\tilde{a}_{1}\right|, \tilde{a}_{N}\right)>>1$. Let us normalize these numbers dividing them by $2 \tilde{a}: a_{i}=\tilde{a}_{i} / 2 \tilde{a} \in[-1 / 2,+1 / 2]$. It is clear that the solution of the problem (1) is the same when we use initial numbers $\tilde{a}_{i}$ or normalized numbers $a_{i}$. The last numbers can be presented in the form (9), if we set $\alpha_{i}=a_{i}+\frac{1}{2}$ and $t=\frac{1}{2}$. From our argumentation it follows that the numbers $a_{i}$ can always be presented in the form (9). Then the following statement is right (the proof see in the Appendix).

Theorem. When $t$ increasing from the initial value $t=0$, the ground state sequentially coincides with the vectors $\mathbf{s}(k)(6)$ in the following order:

$$
\mathbf{s}(0) \rightarrow \mathbf{s}(1) \rightarrow \ldots \rightarrow \mathbf{s}(k-1) \rightarrow \mathbf{s}(k) \rightarrow \ldots \rightarrow \mathbf{s}(n-1) \rightarrow \mathbf{s}(n) .
$$

The jump of the ground state $\mathbf{s}(k-1) \rightarrow \mathbf{s}(k)$ occurs when $t$ transfers through the critical value:

$$
t_{k}=\frac{\hat{\alpha}-2 \hat{\alpha}_{k}+(N-2 k+2) \alpha_{k}}{2(N-2 k+1)}, \quad k=1,2 . ., n,
$$

where analogously of Eq.(7) $\hat{\alpha}=\sum_{i=1}^{N} \alpha_{i}$ and $\hat{\alpha}_{k}=\sum_{i=1}^{k} \alpha_{i}$. When $t$ belongs to the interval $\left[t_{k}, t_{k+1}\right]$, the ground state of the functional is the configuration $\mathbf{s}(k)$.

This theorem generalizes the previous results obtained in [6]. The theorem describes exhaustively the behavior of the ground state for the problem (1)-(2). Depending on the values of external parameters $\left\{a_{i}\right\}$ each of the configurations $\mathbf{s}(k), k=0,1, \ldots, n$ can turn out to be the ground state of the functional. For $t$ from the interval $\left[t_{k}, t_{k+1}\right]$ the energy of the ground state is the linear function of the parameter $t$. It can be easily seen from the expressions (8) substituting the values $a_{i}$ in the form (9):

$$
E_{k}(t)=A_{k}+t \cdot B_{k}, \quad k=0,1, \ldots, n,
$$

where up to the factor $1 / 2 N$ we have:

$$
A_{k}=\hat{\alpha}-(N-2 k)\left(\hat{\alpha}-2 \hat{\alpha}_{k}\right), \quad B_{k}=(N-2 k)^{2}-N .
$$



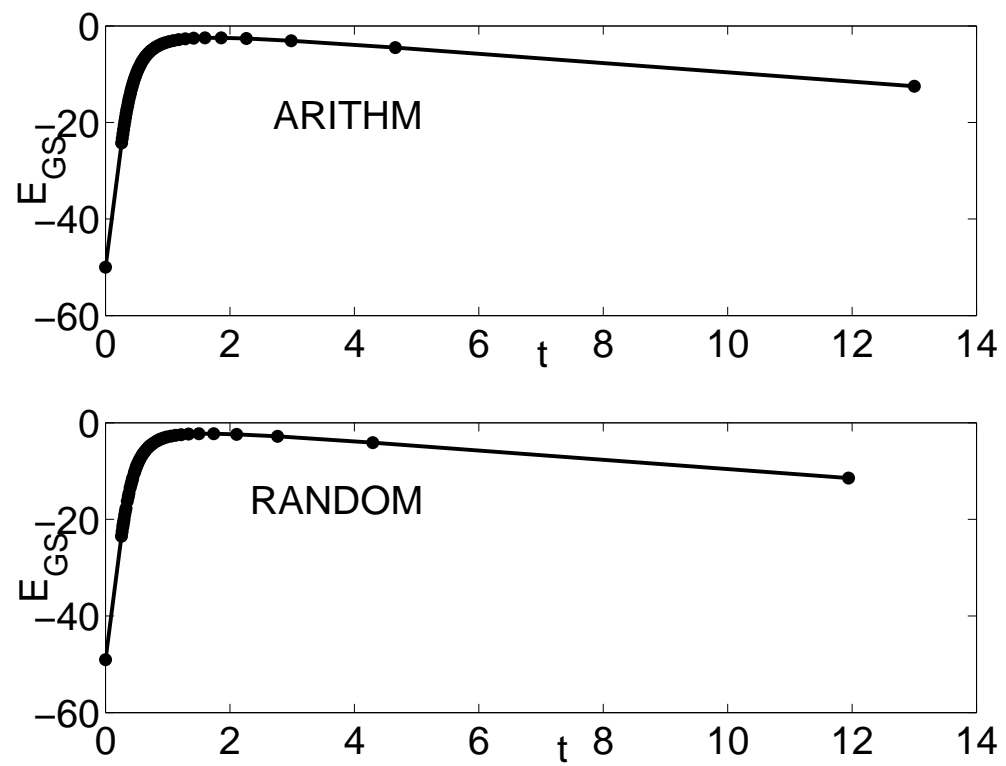

Fig. 1. The dependence of the ground state energy $E_{G S}$ on the parameter $t$ for additive matrices of the dimensionality $N=100$ : the upper one is the arithmetical additive matrix, and the lower one is the random matrix (see the body of the text). The values $E_{G S}$ for the points $t_{k}$ are marked. 
In Fig. 1 for $N=100$ it is shown how the energy of the ground state depends on the parameter $t$. The upper panel corresponds to the case when the values $\alpha_{i}$ constitute the arithmetical progression: $\alpha_{i}=i / N, \quad i=1,2, . ., N$. On the lower panel the analogous plot is shown for a random additive matrix, when $\alpha_{i}$ are random numbers from the interval $[0,1]$. Along the abscissa axis the values of the parameter $t$ are shown, along the axis of ordinates we show the energy of the ground state calculated in the points $t_{k}$ (11). The first value of the parameter $t$ for which the energy of the ground state is calculated is equal to zero: $t_{0}=0$. Since both plots are very similar, we analyze only one of them; for example the upper one.

We see that the energy of the ground state is nontrivially depended on the parameter $t$. For small values, $t \sim 0$, very deep minima correspond to the ground state. Then, when $t$ increases, the depth of the global minimum decreases very quickly and it reaches a minimal value when $t \approx 2$. For these values of $t$ all matrix elements become negative. During further increase of $t$ the depth of the global minimum slowly but steadily increases. It becomes deeper and deeper. Which properties of the energy surface reflect non-monotone change of the depth of the global minimum? What properties are responsible for its minimal depth? For the time being we cannot answer these questions. Using formulae (10)-(13) everyone can be certain of universal character of the curves shown in Fig.1.

Up till now we can neither extend these results onto local minima of the functional, nor obtain analytical description of other interesting characteristics such as the number of different minima, distribution of local minima with respect to their depths and distances to the ground state and so on. However, if the ground state is known, these characteristics can be studied with the aid of computer simulations. Now we turn to presentation of these results.

\section{Computer Simulation}

For given $N$ and $\left\{\alpha_{i}\right\}$ for each value of $t$ we generated an additive matrix. We did $10^{5}$ random starts (see Introduction) and obtained the same number of local minima. For each minimum we fixed its depth (the energy $E_{l}$ ), the relative Hamming distance $D_{l}$ between the minimum and the ground state and other characteristics. Thus as a result of a great number of random trials for each value of $t$ we could estimate: a) the probability of random finding of the ground state $\left.p_{G S} ; \mathrm{b}\right)$ the deepest of the obtained minimum and the distance from it to the ground state; c) the number of different minima $K$, their distribution over energies and distances from the ground state and so on. The parameter $t$ was varied from zero up to the maximal value $t_{n}$. For two dimensionalities $N=100$ and $N=1000$ such experiments were done for both arithmetical and random additive matrices.

In Fig. 2 for the arithmetical additive matrix of dimensionality $N=100$ the dependence of some of the listed characteristics on the parameter $t$ is shown. Let us explain what the graphs shown on different panels of the figure mean.

On the upper panel the probability to find the ground state $p_{G S}$ is shown. We see that in the region of small values of $t(t<1.8)$, where the depth of the global minimum is large, the probability to find the ground state is notably different from zero. On the 


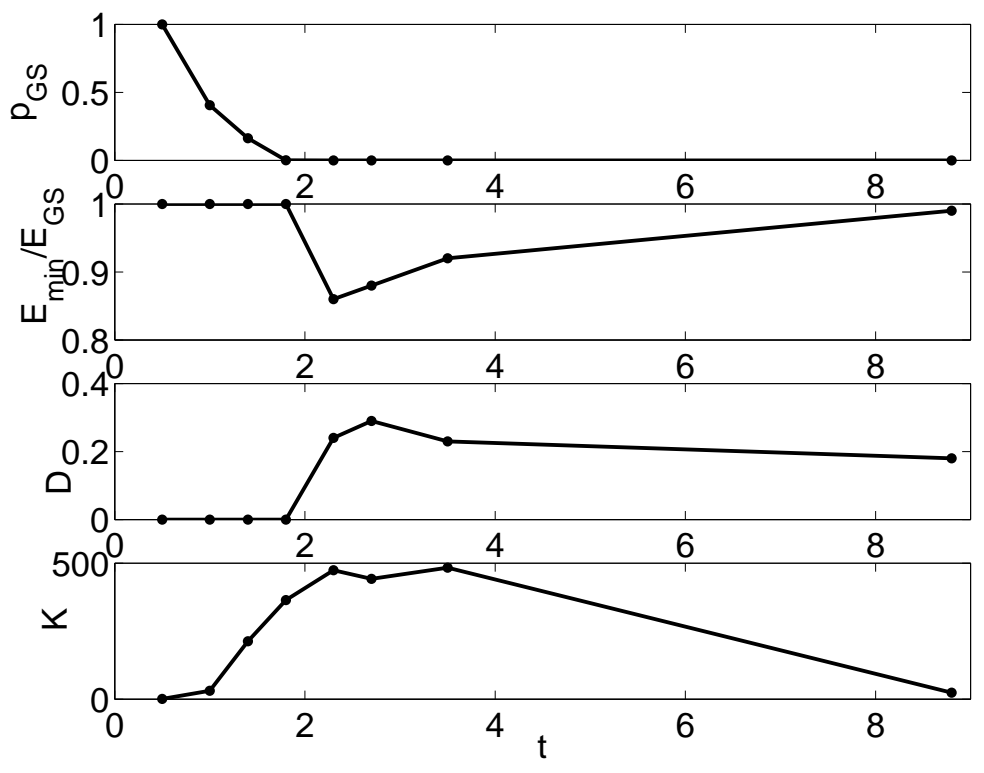

Fig. 2. For arithmetical additive matrix of dimensionality $N=100$ the following graphs are shown: on the upper panel is the probability to find the ground state; on the next panel is the ratio of depth of the deepest found minimum to the depth of the global minimum; on the next panel is the relative Hamming distance between the deepest minimum and the ground state; on the bottom panel is the number of different energies of local minima. 
contrary, in the region of large values of $t$, where the global minimum becomes rather shallow, the probability to find it is equal to zero (it is less than $10^{-5}$ ). At the same time it is not important which configuration $\mathbf{s}(k)$ is the ground state.

Apparently, such behavior of the probability $p_{G S}$ is one more confirmation of the law, which was theoretically predicted in [3], [4]: the deeper minimum, the greater probability to find it under the random search.

For the matrix of dimensionality $N=1000$ the behavior of the given characteristic is an analogous one. The value of the parameter $t$ for which the probability to find the ground state becomes zero, increases up to the value $t \approx 4$.

On the second panel from the top the ratio of the found deepest minimum $E_{\min }$ to the global minimum $E_{G S}, E_{\min } / E_{G S}$, is shown. This ratio takes on a value from the interval $[0,1]$. At first, while the ground state still can be found, this ratio is equal to 1 . Then in the region of the values $t \approx 2.3-2.5$ this characteristic has a sharp downward excursion, which soon changes to a steady increasing and tends to 1 asymptotically. The minimal value of this characteristic is $E_{\min } / E_{G S} \approx 0.85$. It shows that in the worst case the objective function is $15 \%$ less than the optimal value.

For matrices of dimensionality $N=1000$ the behavior of the ratio $E_{\min } / E_{G S}$ is absolutely analogous. The deepest downward excursion of the graph takes place when $t \approx 4$, and its depth increases noticeably: the minimal value of the ratio is equal $E_{\min } / E_{G S} \approx 0.5$. In other words, when the dimensionality of the problem increases the found suboptimal solution will be worse comparing with the global minimum.

Note, that for the large values of $t \sim 7-8$, when the ratio $E_{\min } / E_{G S}$ is close to 1 , the probability to find the ground state as before is equal to 0 . The same also takes place in the case $N=1000$.

On the second panel from the bottom it is shown how the distance $D$ between the deepest local minimum and the ground state depends on the parameter $t$. (By the distance we understand the relative Hamming distance $D=\left(N-a b s\left(\mathbf{s}, \mathbf{s}^{\prime}\right)\right) / 2 N \in$ $[0,0.5]$.)

At first, while the ground state still can be found this distance is equal to 0 (see the beginning of the graph). Then in the interval of the "worst" values of $t$ the distance $D$ increases sharply up to the value $D_{\max } \approx 0.3$. After that the distance between the deepest local minimum and the ground state is stabilized near the value $D=0.2$. Let us add that for additive matrices of dimensionality $N=1000$ suboptimal solution is far away from the ground state. This distance is $D \approx 0.4$.

The general conclusion is as follows: for rather large values of $t$, when as a result of the random search it is possible to find suboptimal solution only, this solution is sufficiently far from the ground state. However, the ratio of the minima depths $E_{\min } / E_{G S}$ can be of order of 1 (in Fig.2 this situation corresponds to the values of $t>4$ ). This combination of properties is possible only if the energy surface consists of a large number of local minima, which depths not strongly differ one from each other and from the global minimum. We may conclude, that for large values of $t$, when elements of connection matrix are large negative numbers, the construction of the energy surface is as aforesaid.

On the bottom panel we show the dependence of the number of different energies of local minima $K$ on the value of the parameter $t$. As a rule each energy is many times 
degenerated. To estimate the number of different local minima it is necessary to analyze how many different configurations correspond to the same energy. Nevertheless, such characteristic as the number of energy levels is also of interest.

For an arithmetical additive matrix of the dimensionality $N=100$ the maximal value of the characteristic $K$ is reached in the region $t \sim 3$. This maximum is comparatively small, $\sim 500$. However, it turns out that each energy is many times degenerated, and the number of different local minima is an orders of magnitude greater. For a random additive matrix of the same dimensionality the maximal value of the characteristic $K$ is equal to tens of thousands (the graph is not presented).

For the additive matrices of the dimensionality $N=1000$ the general form of the graph of the characteristic $K$ is analogous. In this case the maximal value, $K_{\max } \sim$ $4 \cdot 10^{4}$, is reached in the region $t \approx 5$. Since for each $t$ only $10^{5}$ random starts have been done, this means that each second start leads the system into new local minimum. In other words, for middle values of $t$ the number of local minima is very big.

\section{Discussion and Conclusions}

For additive matrices the method of finding of the global minimum of the quadratic binary functional is pointed out. We propose the $t$-parametrization of additive matrices that allows one to get an exhaustive classification for all variants possible for the ground state.

For not great values of $t$ (let us say for $t \in[0,2]$ ) among matrix elements there are positive as well as negative ones; or all elements are negative, but they are small in modulus. In this case the depth of the global minimum is very big. Here the probability to find the ground state in random search is rather high: $p_{G S} \sim 0.5-1.0$. It can be supposed that in this case the energy surface has a small number of local minima whose depths noticeably less then the depth of the global minimum.

On the contrary, for the great values of $t$ all matrix elements are negative and they are big in modulus. In this case it is practically impossible to find the ground state with the aid of the random minimization, since the probability to get into the global minimum is negligible small. Apparently in this case the energy surface contains very large number of local minima that only slightly differ from each other in depths. Here the global minimum is only insignificantly deeper than local minima. Varying the value of the parameter $t$ it is possible to get over from one type of the energy surface to the other one. So, additive matrices are good models for examining the energy surfaces in the general case.

By this time additive matrices for large values of $t$ can be used for testing of new algorithms of the quadratic binary minimization. Indeed, on the one hand, with the aid of the formulae (10)-(13) the ground state always can be found. On the other hand, for large values of the parameter $t$ it is practically impossible to find the ground state with the aid of the random minimization. 


\section{Acknowledgment}

The work has been done with participation of Anton Pichugin in the framework of the program supported by the grant \#356.2008.9 of President of Russian Federation and in part by Russian Basic Research Foundation (grant \#09-07-00159).

\section{References}

1. Hartmann, A.K., Rieger, H. (Eds.): New optimization algorithms in physics. Wiley-VCH, Berlin, 2004.

2. Junger, M.: The Spin Glass Ground State Server. http://www.informatik.uni-koeln.de/ls_juenger/research/sgs/sgs.html

3. Hertz, J., Krogh, A., Palmer, R.: Introduction to the Theory of Neural Computation. AddisonWesley, NY, 1991.

4. Kryzhanovsky, B.V., Magomedov, B.M., Mikaelyan, A.L.: A Relation between the Depth of a Local Minimum and the Probability of Its Detection in the Generalized Hopfield Model. Doklady Mathematics, 2005, v. 72(3), pp. 986-990.

5. Kryzhanovsky, B.V., Kryzhanovsky, V.M., Mikaelian, A.L.: Binary optimization: A relation between the depth of a local minimum and the probability of its detection. 4th International Conference on Informatics in Control, Automation and Robotics, pp.5-10, ICINCO 2007, Anger, France.

6. Litinskii, L.B.: High-symmetry Hopfield-type neural networks. Theoretical and Mathematical Physics, 1999, v.118(1), pp. 107-127.

\section{Appendix}

In the beginning of Section 2 it was shown that only one of configuration vectors $\mathbf{s}(k)$ (6), $k=0,1, \ldots, n=[N / 2]$ can be the ground state. Using the representation (9) of $a_{i}$ it is easy to obtain Eq. (12) for the energies of $\mathbf{s}(k)$-configurations: $E_{k}(t)=A_{k}+t B_{k}$, where $A_{k}$ and $B_{k}$ are given by Eq.(13). As functions of the parameter $t$ energies $E_{k}(t)$ are straight lines. We have to analyze the behavior of the set $\left\{E_{k}(t)\right\}_{0}^{n}$. When a straight line $E_{l}(t)$ is lower all other straight lines, the configuration $\mathbf{s}(l)$ is the ground state.

For simplicity we restrict ourselves to the case of even $N=2 n$. Let us write down the expression (13) in more details:

$$
\begin{gathered}
A_{0}=-(N-1) \hat{\alpha}<A_{1}<\ldots<A_{n}=\hat{\alpha} \\
B_{0}=(N-1) N>B_{1}>\ldots>B_{n}=-N
\end{gathered}
$$

When $k$ increasing, the free term $A_{k}$ of the straight line $E_{k}(t)$ increases monotonically. In other words, the intersection of the straight line with ordinate axis rises higher and higher. On the other hand, when $k$ increasing the coefficient $B_{k}$ decreases monotonically, so that in the end it even becomes negative. For the case $N=6$ the typical behavior of the set of straight lines $\left\{E_{k}(t)\right\}_{0}^{n}$ is shown in Fig.3. We use this figure to explain how the ground state depends on the parameter $t$.

When $t=0$ all the matrix elements are positive and the configuration $\mathbf{s}(0)=$ $(1, \ldots 1)$ is the ground state. Let us increase $t$ little by little. At first the straight line 
$E_{0}(t)$ is lower than all other straight lines. Consequently, $\mathbf{s}(0)$ remains the ground state. Than for some value of the parameter $t$ the straight line $E_{0}(t)$ is intersected by another straight line. After that this straight line turns out to be lower than all other straight lines. Taking into account the relations (A1) it is easy to see that the first straight line that intersects $E_{0}(t)$ is $E_{1}(t)$ (see also Fig.3). After this intersection the configuration $\mathbf{s}(1)$ becomes the ground state. It is the ground state until another straight line intersects the straight line $E_{1}(t)$. After that this straight line turns out to be lower than all other straight lines. From the aforesaid argumentation it is evident that it will be the straight line $E_{2}(t)$ (see Fig.3). Then the configuration $\mathbf{s}(2)$ will be the ground state, and so on. It can be shown that if the straight line $E_{k-1}(t)$ is lower than all other straight lines, the first straight line that intersects $E_{k-1}(t)$ is $E_{k}(t)$. The intersection takes place in the point $t_{k}$ (11) that is the solution of equation $A_{k-1}+t \cdot B_{k-1}=A_{k}+t \cdot B_{k}$.

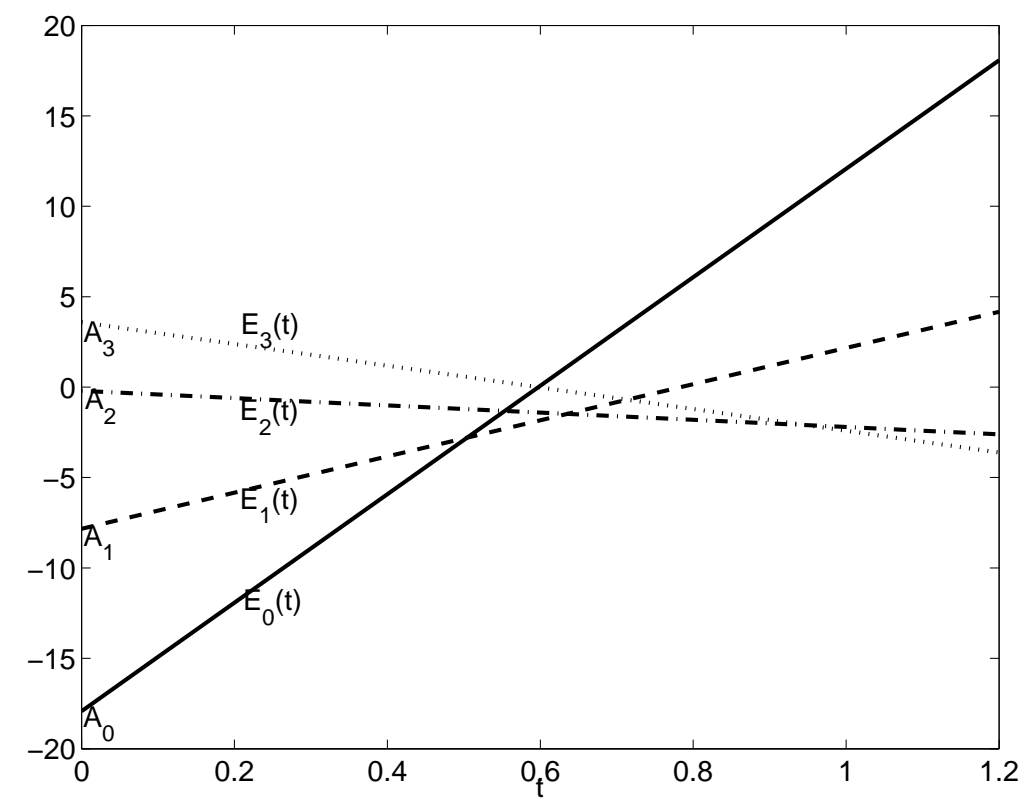

Fig. 3. For random additive matrix of dimensionality $N=6$ the straight lines $E_{k}(t)=$ $A_{k}+t \cdot B_{k}$ are shown for $k=0,1,2,3$ (see the body of the text). 